\title{
Unified Drain Current Model of Armchair Graphene Nanoribbons with Uniaxial Strain and Quantum Effect
}

\author{
EngSiew Kang ${ }^{1,2}$ and Razali Ismail ${ }^{2}$ \\ ${ }^{1}$ Department of Electrical \& Electronic, Southern University College, 81300 Skudai, Johor, Malaysia \\ ${ }^{2}$ Faculty of Electrical Engineering, Universiti Teknologi Malaysia, 81300 Skudai, Johor, Malaysia
}

Correspondence should be addressed to Razali Ismail; razali@fke.utm.my

Received 8 January 2014; Revised 31 March 2014; Accepted 31 March 2014; Published 23 April 2014

Academic Editor: Yingkui Yang

Copyright (C) 2014 E. Kang and R. Ismail. This is an open access article distributed under the Creative Commons Attribution License, which permits unrestricted use, distribution, and reproduction in any medium, provided the original work is properly cited.

\begin{abstract}
A unified current-voltage $I-V$ model of uniaxial strained armchair graphene nanoribbons (AGNRs) incorporating quantum confinement effects is presented in this paper. The $I-V$ model is enhanced by integrating both linear and saturation regions into a unified and precise model of AGNRs. The derivation originates from energy dispersion throughout the entire Brillouin zone of uniaxial strained AGNRs based on the tight-binding approximation. Our results reveal the modification of the energy band gap, carrier density, and drain current upon strain. The effects of quantum confinement were investigated in terms of the quantum capacitance calculated from the broadening density of states. The results show that quantum effect is greatly dependent on the magnitude of applied strain, gate voltage, channel length, and oxide thickness. The discrepancies between the classical calculation and quantum calculation were also measured and it has been found to be as high as $19 \%$ drive current loss due to the quantum confinement. Our finding which is in good agreement with the published data provides significant insight into the device performance of uniaxial strained AGNRs in nanoelectronic applications.
\end{abstract}

\section{Introduction}

As projected by Moore's law, the semiconductor industry is experiencing an exponential growth in the number of transistors on chip over the past four decades. In conjunction with Moore's law, International Technology Roadmap for Semiconductors (ITRS) predicted that the size of the gate length is to be eventually shrunk into $4.5 \mathrm{~nm}$ in 2023 . However, the aggressive geometry scaling of the complimentary metal-oxide-semiconductor (CMOS) to stimulate the performance enhancement of Si based devices is currently approaching its fundamental limits. As CMOS device is scaled down to $50 \mathrm{~nm}$ gate length, serious degradation of carrier effective mobility becomes aggravated which is attributed to an increased channel doping to suppress short channel effect and higher effective field [1]. Hence, it is critical to develop a new generation of nanoscale transistors that can uphold a better performance than that of the state-of-theart MOS field-effect transistor (FET), such as FinFets, carbon nanotubes (CNT), silicon nanowire, and recently graphene nanoribbons FET. Among them, graphene-based devices are no doubt the promising alternatives in sustaining the progress in nanotechnology [2].

Graphene possesses various fascinating electrical and physical properties, such as an extremely high mobility of the charge carrier, high switching speed with ballistic transport behaviours, and anomalous quantum Hall effects [3, 4]. These excellent electronic properties of graphene make it a promising alternative as the building block in potential nanoelectronic devices. However, graphene shows gapless nature and therefore are unsuitable for logic applications. Graphene patterned into nanoribbons referred to as graphene nanoribbons (GNRs) has been demonstrated to possess band gap opening made possible by tuning the ribbon width $[5,6]$. To further increase the GNRs application as FET, various studies have attempted to modulate the electronics structure using mechanical deformation $[3,7,8]$. This offers a tempting 
prospect of controlling the electronic properties of GNRs structure by the introduction of uniaxial strain.

Theoretically, the potential of uniaxial strain on the energy gap of GNRs has been widely adopted based on the $a b$ initio approaches and tight-binding approximation [9-11]. It has been shown that zigzag GNRs (ZGNRs) and armchair GNRs (AGNRs) possess distinct energy gap properties under strain. Despite the fact that there have been many studies on the strain effect in AGNRs, most of the previous works focused on the electronic band structure, while the effect of strain on the drain current has seldom been studied. As the carrier transport properties in AGNRs has strong relation with the electronic band structure, it is mandatory to investigate the strain effect on the drain current for performance metric evaluation. Furthermore, in order to fully understand the transport properties, it is also important to understand the density of carriers around the Dirac point as well as the scattering of the carriers by the impurities [12], which is related to the quantum confinement effects. It has been recognized that the classical idea of capacitance needs to be modified at the nanoscale. However, there are very few investigations of the confinement properties that have been carried out, particularly on uniaxial strained AGNRs.

Therefore in this paper, the quantum confinement effect is taken into consideration in developing the current-voltage $(I-V)$ characteristic. In addition, the $I-V$ model is enhanced by integrating both linear and saturation regions into a unified $I-V$ model of uniaxial strained AGNRs. The unified $I-V$ model adopts the energy dispersion based on tight-binding approximation and the analytical results were compared against experimental data. The comparison for a set of similar parameters shows excellent agreement, indicating the accuracy of the proposed model.

\section{Analytical Quantum Capacitance}

The quantum behaviours of AGNRs under uniaxial strain were studied by self-consistently solving the energy band structure in an atomistic basic set based on tight-binding approximation. The energy dispersion throughout the entire Brillouin zone of AGNRs is given by [14]:

$$
\begin{aligned}
E(k)= \pm[ & t_{1}^{2}+4 t_{2}^{2} \cos ^{2}\left(\frac{p \pi}{m+1}\right) \\
& \left.+4 t_{1} t_{2} \cos \left(\frac{p \pi}{m+1}\right) \cos \left(\frac{3}{2} k_{x} a_{\mathrm{cc}}\right)\right]^{1 / 2}
\end{aligned}
$$

where $t_{1}=t_{0} /(1+\varepsilon)^{2}$ and $t_{2}=t_{0} /(1+\varepsilon / 4)$ with $\varepsilon$ representing the magnitude of uniaxial strain and $t_{0}=$ $-2.7 \mathrm{eV}$ is the nearest carbon-carbon $(\mathrm{C}-\mathrm{C})$ tight binding overlap energy without strain, $a_{\mathrm{cc}}=1.42 \AA$ is the $\mathrm{C}-\mathrm{C}$ bond length, $k_{x}$ is the wave vector component along the $x$ direction, $m$ is the number of dimmer lines across the ribbon width, and $p$ is the band index running from 1 to $n$. The width of AGNRs, $w$, is proportional to $m$ given by the expression [15]:

$$
w=(m-1) \frac{\sqrt{3}}{2} a_{\mathrm{cc}}
$$

Equation (1) shows that the energy dispersion relation in uniaxial strained AGNRs obeys the time-reversal symmetry. As a result, the energy separation between the conduction band top and valence band top occurs at $\Gamma$ points $\left(k_{x}=0\right.$, central Brillouin zone) [16]. Thus, energy band gap can be derived as

$$
E_{g}=2\left[t_{1}+2 t_{2} \cos \left(\frac{p \pi}{m+1}\right)\right] .
$$

By using the first order trigonometry function, (1) can be further simplified into the following equation:

$$
E(k)= \pm \sqrt{\frac{E_{g}^{2}}{2}+B k_{x}^{2}}
$$

where

$$
B=-\frac{9}{2} t_{1} t_{2} \cos \left(\frac{p \pi}{m+1}\right) a_{\mathrm{cc}}^{2}
$$

The band structure in (4) is indeed a nonparabolic relation with the wave vector. However, for low-lying energy states where most of the carriers likely reside, the band structure can be approximated to parabolic characteristic. By applying the square root approximation model, $\sqrt{1+\alpha^{2}} \approx$ $1+\alpha^{2} / 2$, the parabolic band energy in low energy limit is obtained as

$$
E(k)=\frac{E_{g}}{2}-\frac{B k_{x}^{2}}{E_{g}} .
$$

In one-dimensional nanodevice, the density of states (DOS) reveals the probability of the available states to be occupied. By including the effect of electron spin using (6), the DOS of AGNRs under uniaxial strain can be expressed as

$$
D(E)=\frac{\Delta N_{x}}{\Delta E L}=\frac{1}{4 \pi} \sqrt{\frac{2 E_{g}}{B\left(2 E-E_{g}\right)}},
$$

where $N_{x}$ is the quantum number and $L$ is the ribbon length.

The number of electrons and holes per cubic centimetre with energies between $E$ and $(E+d E)$ has been established to be $D(E) f(E) d E$ and $D(E)[1-f(E)] d E$. The total carrier density in the channel is formally given as

$$
n=\int_{E_{c}}^{E_{\text {top }}} D(E) f(E) d E,
$$

where $f(E)=1 / 1+e^{E-E_{F} / k_{B} T}$ is the Fermi Dirac distribution which describes the degeneracy nature of the electron concentration. Here, $E_{F}$ is the Fermi energy, $k_{B}$ is the Boltzmann constant, and $T$ is the ambient temperature in Kelvin. $E_{\text {top }}$ and $E_{c}$ are the top and bottom conduction band, respectively. By substituting the normalized Fermi energy as $\eta=\left(E_{F}-\right.$ $\left.E_{g} / 2\right) / k_{B} T$ and transforming $E$ to $x=\left(E-E_{g} / 2\right) / k_{B} T$ and $d x k_{B} T=d E$, the total carrier density inside the channel can be simplified into

$$
n=\frac{1}{4 \sqrt{\pi}} \sqrt{\frac{E_{g} k_{B} T}{B}} \Im_{-1 / 2}(\eta) .
$$


The expression of the quantum capacitance per unit length, $C_{Q}$, in AGNRs under uniaxial strain is determined by the derivative of the charge density:

$$
C_{\mathrm{Q}}=\frac{d Q}{d V}=q^{2} \frac{d n}{d E},
$$

where $d Q=q d n$ is the charge differential and $d V=d E / q$ is the voltage differential for the applied voltage with $q$ as the electronic charge. The quantum capacitance can be calculated as

$$
C_{Q}=\frac{e^{2}}{4 \pi \sqrt{k_{B} T}} \frac{\sqrt{E_{g}}}{\sqrt{B}} x^{-1 / 2} e^{\eta} .
$$

The equivalent gate capacitance can be interpreted as a series combination of $C_{Q}$ and insulator capacitance, $C_{\text {ins }}$. The $C_{\text {ins }}$ for AGNRs can be obtained and calculated by the following simple expression [17]:

$$
C_{\text {ins }}=N_{G} \kappa \varepsilon_{0}\left(\frac{W}{t_{\text {ins }}}+\alpha\right),
$$

where $N_{G}$ is the number of gates ( 1 for the single gate geometry and 2 for double gate geometry), $\kappa$ is the relative dielectric constant of the gate insulator, $t_{\text {ins }}$ is the thickness of the gate insulator, $W$ is the ribbon width, and $\alpha \simeq 1$ is a dimensionless fitting parameter.

\section{Unified Drain Current Modelling}

An accurate and precise current-voltage $\left(I_{D}-V_{D}\right)$ characteristic of uniaxial strained AGNRs can be obtained by including the quantum capacitance due to the quantum confinement effect. In general, the drain current, $I_{D}$, as a function of the drain voltage, $V_{D}$, and gate voltage, $V_{G S}$ is given by

$$
\begin{array}{r}
I_{D}=\frac{\mu_{\mathrm{effi}} C_{G}}{2 L}\left[\frac{2\left(V_{G S}-V_{T}\right) V_{D}-V_{D}^{2}}{1+V_{D} / V_{C}}\right] \\
\text { for } 0 \leq V_{D} \leq V_{D \text { sat }},
\end{array}
$$

where $V_{T}$ is the threshold voltage and $V_{D \text { sat }}$ is the drain voltage at which the drain carrier concentration becomes maximum, consistent with the drain saturation current [18]. The critical voltage, $V_{C}$, is given as

$$
V_{C}=L E_{C}=L \frac{v_{\text {sat }}}{\mu_{\text {eff }}},
$$

where $v_{\text {sat }}$ is the velocity saturation and $\mu_{\text {eff }}$ is the low field mobility that is degraded by the gate electric field and ballistic channel effects.

All the carriers in the channel travel at the saturation velocity by the onset of the current saturation, where the electric field is extremely high. The saturation current, $I_{D \text { sat }}$, is given by the following:

$$
I_{D \text { sat }}=C_{G}\left(V_{G S}-V_{T}-V_{D \text { sat }}\right) v_{\text {sat }} \text { for } V_{D} \geq V_{D \text { sat }} .
$$

Equations (13) and (15) must reconcile at the onset of current saturation. This reconciliation gives $V_{D \text { sat }}$ and $I_{D \text { sat }}$ the following expressions:

$$
\begin{gathered}
V_{D \text { sat }}=V_{C}\left[\sqrt{1+\frac{2\left(V_{G S}-V_{T}\right)}{V_{C}}}-1\right], \\
I_{D \text { sat }}=\frac{1}{2} \frac{C_{G} \mu_{\text {eff }}}{L} V_{D \text { sat. }}^{2} .
\end{gathered}
$$

The drain current can be expressed as a second order function of $V_{D \text { sat }}$ as given by [19]

$$
I_{D}=\frac{\mu_{\mathrm{eff}} C_{G}}{2 L}\left[\frac{2\left(V_{G S}-V_{T}\right) V_{D \text { sat }}-\beta V_{D \text { sat }}^{2}}{1+V_{D \text { sat }} / V_{C}}\right],
$$

where $\beta$ is the body-effect coefficient.

By considering the effect of source and drain series resistance, $R_{S D}, I_{D \text { sat }}$ can be obtained as

$$
\begin{aligned}
I_{D \text { sat }}=C_{G}[ & \left(V_{G}-I_{D \text { sat }} R_{S}-V_{T}\right) \\
& \left.-\beta\left(V_{D \text { sat }}-I_{D \text { sat }}\left(R_{S}+R_{D}\right)\right)\right] v_{\text {sat }} .
\end{aligned}
$$

After some algebra,

$$
I_{D \text { sat }}=\frac{C_{G}\left(V_{G S}-V_{T}-\beta V_{D \text { sat }}\right) v_{\text {sat }}}{1-C_{G} v_{\text {sat }}\left[(\beta-1) R_{S}+\beta R_{D}\right]} .
$$

This newly derived $I_{D \text { sat }}$ in (20) should reconcile with the one in the linear region. The $V_{D \text { sat }}$ can then be computed as [19]

$$
V_{D \text { sat }}=\frac{-b-\sqrt{b^{2}-4 a c}}{2 a}
$$

where

$$
\begin{gathered}
a=v_{\mathrm{sat}} C_{G} m R_{S}, \\
b=-\left[V_{G S}-V_{T}+v_{\text {sat }} C_{G}\left(V_{G S}-V_{T}\right)\left(2 R_{S}+m R_{S D}\right)\right. \\
\left.+m E_{\text {sat }} L_{\text {eff }}\right], \\
c=\left(V_{G S}-V_{T}\right) E_{\text {sat }} L_{\text {eff }}+2 v_{\text {sat }} C_{G} R_{S D}\left(V_{G S}-V_{T}\right)^{2} .
\end{gathered}
$$

In order to achieve a smooth transition from linear to saturation, a smoothing function is introduced [20] which can be expressed as

$$
\begin{aligned}
V_{D \text { eff }}=V_{D \text { sat }}-\frac{1}{2}[ & V_{D \text { sat }}-V_{D S}-\delta \\
& \left.+\sqrt{\left(V_{D \text { sat }}-V_{D S}-\delta\right)^{2}+4 \delta V_{D \text { sat }}}\right],
\end{aligned}
$$

where $\delta$ is a fitting parameter [19]. Equation (23) is used to replace $V_{D \text { sat }}$ in (18). Hence, the unified one region equation can be obtained as

$$
I_{D}=\frac{\mu_{\mathrm{eff}} C_{G}}{2 L}\left[\frac{2\left(V_{G S}-V_{T}\right) V_{D \text { eff }}-V_{D \mathrm{eff}}^{2}}{1+V_{D \mathrm{eff}} / V_{C}}\right] .
$$




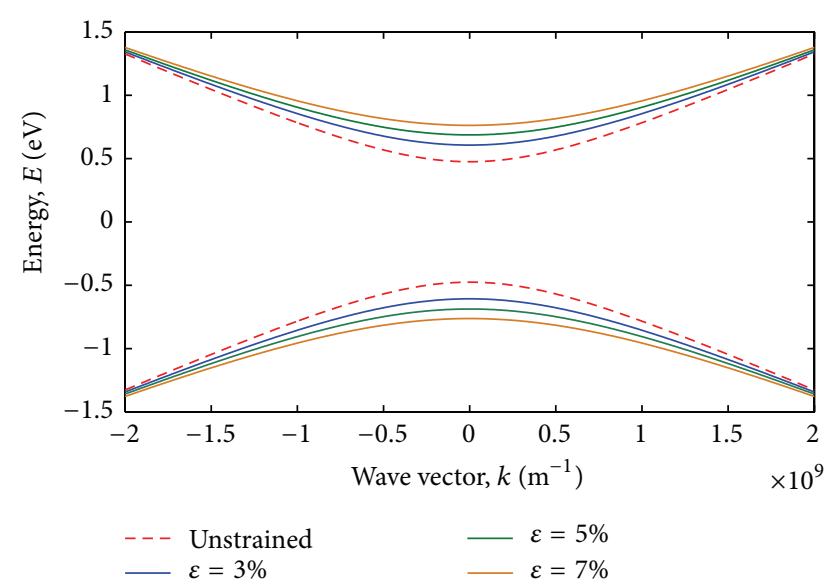

FIGURE 1: Energy band structure of AGNRs under different magnitude of uniaxial strain.

In our preliminary work, it has been demonstrated that the $I_{D}-V_{D}$ characteristics consist of separate linear and saturation regions [21]. The formulation in (24) offers a simplified approach that integrates both regions into a unified $I_{D}-V_{D}$ model of AGNRs

\section{Results and Discussion}

In order to achieve a better understanding of the effect of uniaxial strain on the atomic behaviour of AGNRs, the energy band structure in response to the Bloch wave vector is illustrated in Figure 1. As presented, there is no Fermi crossing along the edges of the Brillouin zone which indicates the emergence of band gap opening in AGNRs. One can observed that the size of the band gap increases almost linearly with the magnitude of strain. The calculated values of $E_{g}$ are $1.21 \mathrm{eV}, 1.37 \mathrm{eV}$, and $1.52 \mathrm{eV}$ which occur at $\varepsilon=3 \%, 5 \%$ and $7 \%$. This phenomenon can be best explained by the shift of the Fermi point perpendicular to the allowed $k$ lines. When a uniaxial strain is applied to the AGNRs channel, the Fermi point deviates from $K$ and thus further shifts the Fermi points [10]. Such sensitive responses of AGNRs to uniaxial strain render them as suitable candidates for the application of strain sensor. The dependence of the energy band gap as a function of the ribbon width is depicted in Figure 2. In general, $E_{g}$ decreases smoothly with the increment in ribbon width due to the weaker confinement in the width direction. A remarkably good agreement of the band gap variation is observed with the experimental data [11], indicating the validity of the tight binding approximation applied in the model.

Figure 3 plots the analytical carrier density as a function of the normalized Fermi energy, $\eta_{F}$, at room temperature for two magnitudes of strain under degenerate condition. While the nondegenerate case has a strict linear curve (in logarithmic scale) with a high slope, the carrier density in this regime has a quasilinear curve and a reduced slope. More precisely, the slope of $\log (n)$ is not constant but gradually decreases with $E_{F}-E_{c} / k_{B} T$.

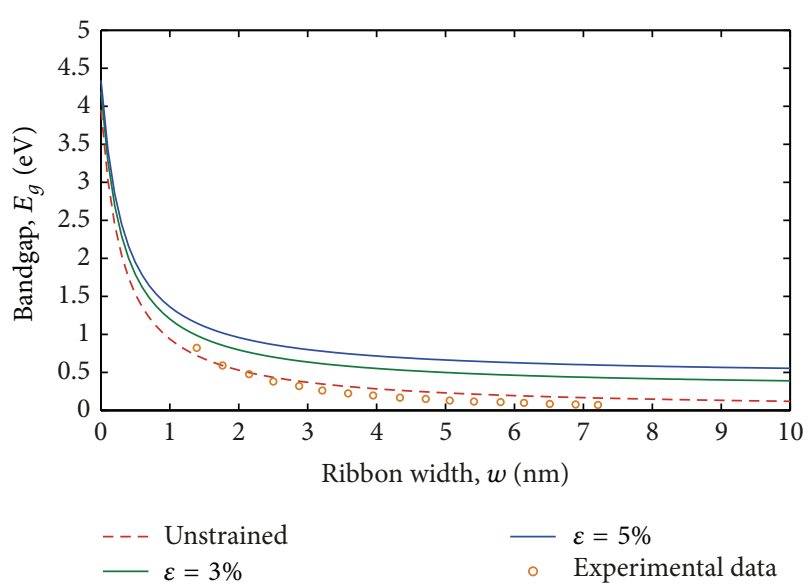

FIGURE 2: The plot of energy band gap as a function of ribbon width for unstrained and strained AGNRs.

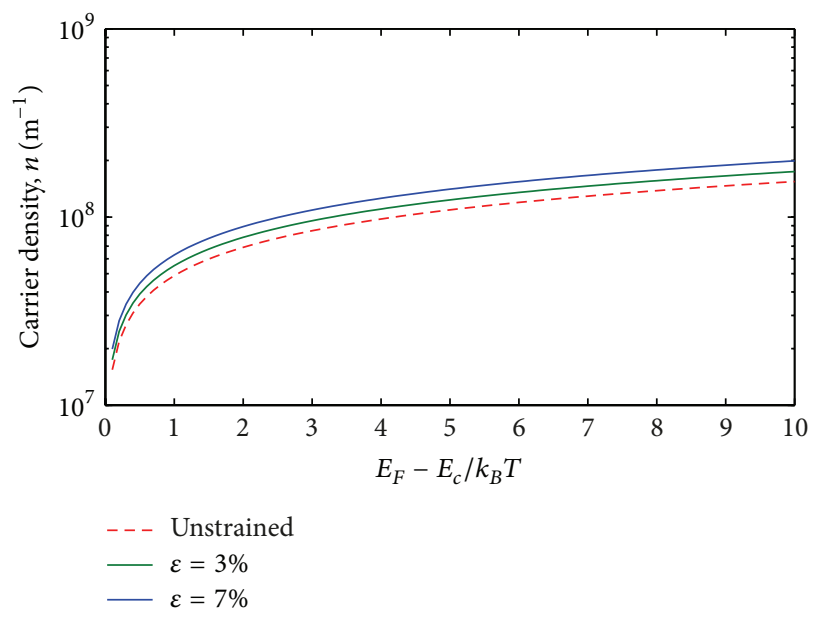

FIGURE 3: The variation of carrier density at room temperature in AGNRs in respect to the normalised Fermi energy for unstrained and strained AGNRs.

Downscaling the device dimensions has stimulated the extensive efforts to further reduce the gate oxide thickness where a strong quantum confinement effect is expected. Based on the energy band structure, the analytical model of quantum capacitance of uniaxial strained AGNRs is derived as in (11) to achieve a better understanding of the atomic behaviours. Figure 4 displays the dependence of quantum capacitance on the strain effect for AGNRs of several ribbon widths. As it can be seen from the plot, quantum capacitance increases linearly with the increment of strain. The obtained small values of quantum capacitance at lower strain are attributed to low DOS characterization of the atomically thin quasi-1D channel [22] and further reduction of the DOS due to quantum confinement boundary conditions in the AGNRs transverse direction. It is also important to notice that the quantum capacitance significantly increases with the decrement in the size of ribbon width which is a direct consequence of energy band gap widening. 


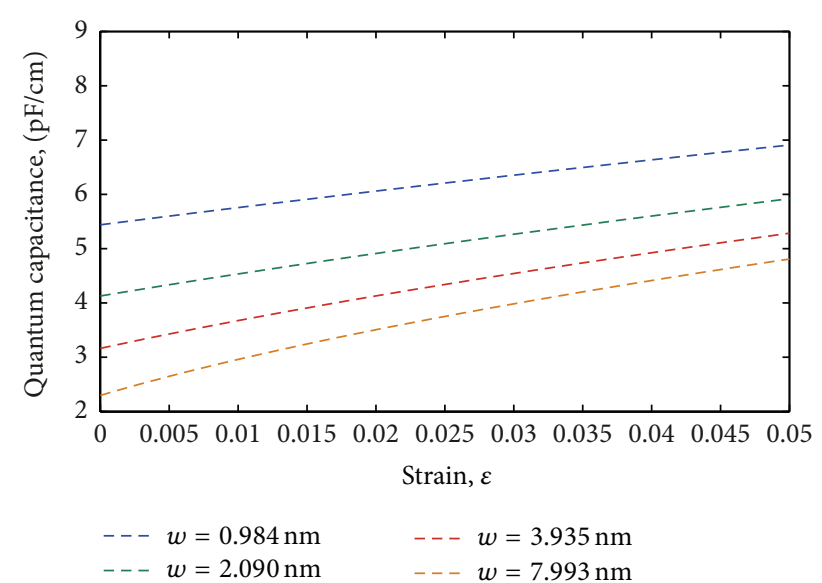

FIGURE 4: The calculated quantum capacitance under uniaxial strain effect for different ribbon widths.

In order to validate the proposed analytical model, the MATLAB simulation results were compared with the experimental data [13] for a range of strain effect as demonstrated in Figure 5. The comparison of the unified $I_{D}-V_{D}$ characteristic between the classical (solid lines) and quantum (dashed lines) for various gate voltages is depicted in Figure 6. Significant drain current reduction in the proposed model is observed, which results from the threshold voltage shift and total gate capacitance degradation due to quantum confinement. It should be noted that the influence of quantum confinement depends on the increment of gate-source voltage. Under $V_{G S}=1.2 \mathrm{~V}$ condition, the drain current for the proposed model drops $1.452 \mu \mathrm{A}$ compared to the classical value. Meanwhile, for $V_{G S}=0.8 \mathrm{~V}$ and $V_{G S}=0.4 \mathrm{~V}$, the current loss is $0.798 \mu \mathrm{A}$ and $0.191 \mu \mathrm{A}$, respectively. This tremendous change may be attributed to the quantum mechanics that introduce an extra energy to pump the electrons to states with higher energy. In the state-of-the-art nanoscale MOSFET, the width of the well is small enough at high electric field so that the carriers' motion is only in the perpendicular direction to the interface. The energy spectrums are then quantized and this gives rise to the splitting of the continuous energy band. Also, the energy levels of these carriers are grouped into several discrete subbands with $2 \mathrm{D}$ density of state. The equally spaced steps show behaviour characteristic of a short channel when $V_{G S}<V_{C}$. On the contrary, for $V_{G S}>V_{C}$, the AGNRs transistor exhibit long-channel behaviour with steps that grow quadratically with the adjusted applied voltage. In fact, if (16) is expanded to second order in $V_{G T}$, one can easily notice the complete absence of pinch off effect [21,23].

Figure 7 shows the performance of the unified $I_{D}-V_{D}$ characteristic at $V_{G S}=0.5 \mathrm{~V}$ with $3 \%$ strain effect, comparing the classical calculation (solid lines) and quantum calculation (dashed). Figure 7(a) shows the result for different channel lengths and Figure $7(\mathrm{~b})$ shows the variation with different oxide thicknesses. In general, the reduction of channel length along with the implementation of ultrathin gate oxide would decrease the channel potential barrier that allows more electron injection from the source to the drain. It is found that

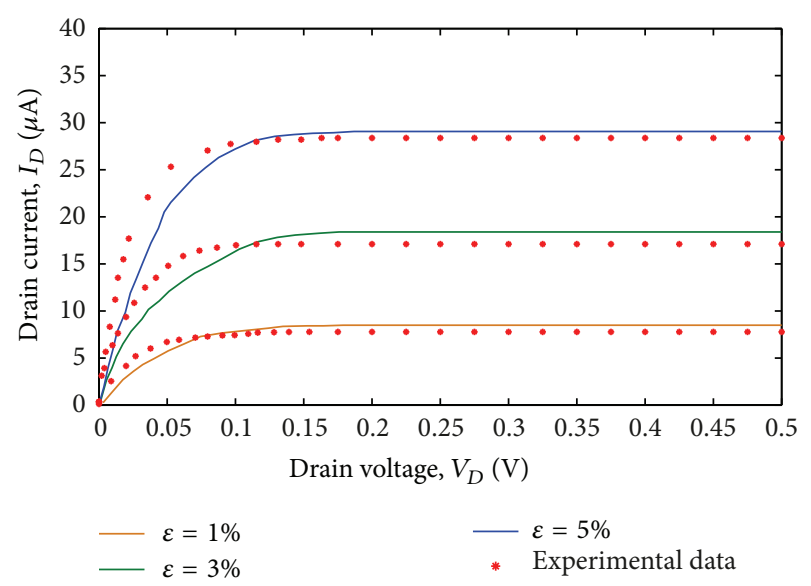

FIGURE 5: The comparison of the $I_{D}-V_{D}$ characteristic of uniaxial strained AGNRs based on the quantum calculation with experimental data [13].

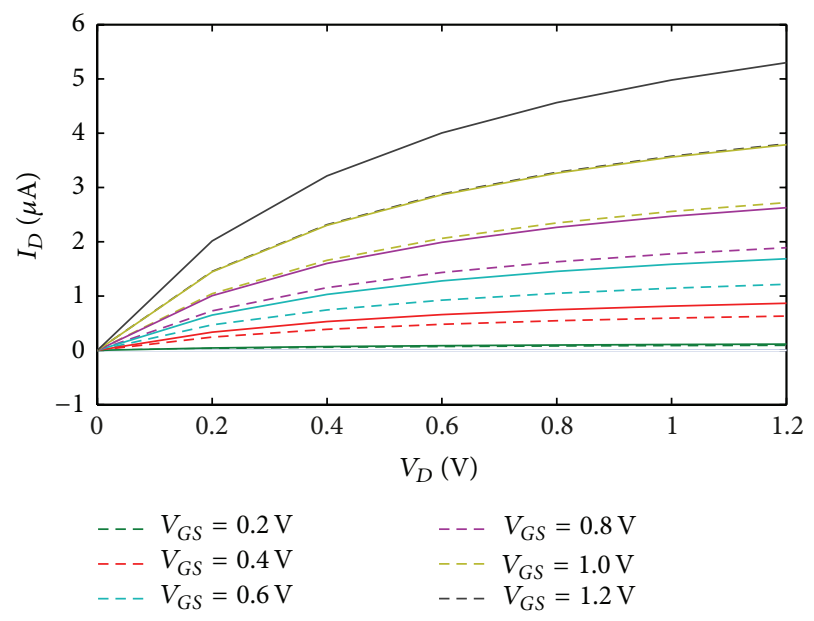

FIGURE 6: $I_{D}-V_{D}$ characteristic of uniaxial strained AGNRs by comparing the classical and quantum approaches. The solid lines are for the classical calculation, while the dashed lines are obtained from the quantum calculation.

when $L=50 \mathrm{~nm}$, larger reduction of drain current due to the quantum confinement can be observed as compared to $L=$ $150 \mathrm{~nm}$ and $L=300 \mathrm{~nm}$. The calculated current loss due to the quantum confinement is $3.579 \mu \mathrm{A}, 1.828 \mu \mathrm{A}$, and $1.219 \mu \mathrm{A}$ for $L=50 \mathrm{~nm}, L=150 \mathrm{~nm}$, and $L=300 \mathrm{~nm}$, respectively. Meanwhile for $T_{\mathrm{OX}}=3 \mathrm{~nm}, T_{\mathrm{OX}}=5 \mathrm{~nm}$, and $T_{\mathrm{OX}}=8 \mathrm{~nm}$, the drain reductions are approximately $2.796 \mu \mathrm{A}, 1.229 \mu \mathrm{A}$, and $0.647 \mu \mathrm{A}$. The presence of energy quantization significantly influences the manner in which electrons respond to the bias. Levitated electron energy levels effectively reduce the inversion charge density, requiring extra gate voltage for the threshold condition [24]. In addition, gate control of the inversion charges is weakened, which is specified by the reduced inversion layer capacitance in the gate capacitance model considering quantization effect. Therefore, significant drain current reduction in the quantum confinement model is observed that results from the threshold voltage shift and 


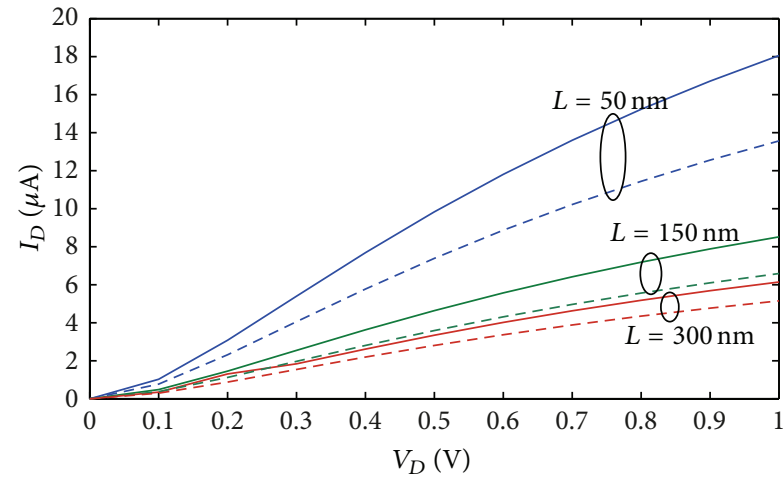

(a)

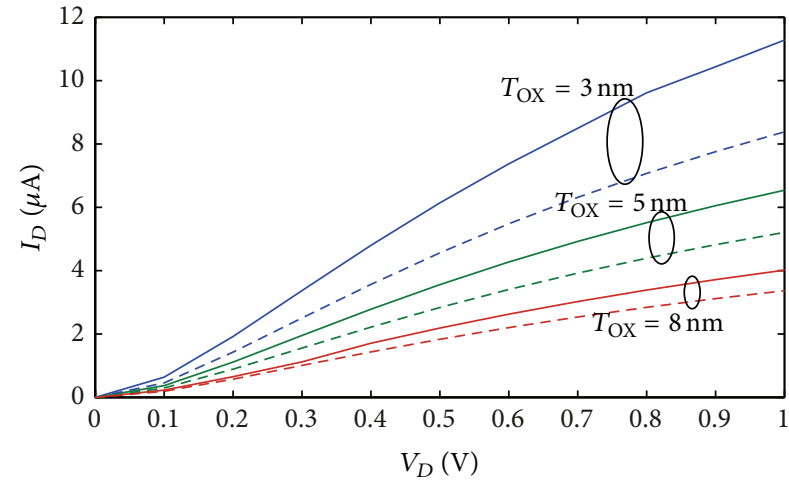

(b)

FIGURE 7: The $I_{D}-V_{D}$ characteristics with classical calculation (solid lines) and quantum calculation (dashed lines) at $V_{G S}=0.5 \mathrm{~V}$ with $3 \%$ strain for three different (a) channel lengths and (b) oxide thicknesses.

gate capacitance degradation. Furthermore, these variations can also be explained by the displacement of the overall carrier distribution towards the substrate [25] attributed to the quantization effect.

Under uniaxial strain, the electronic properties of AGNRs changes dramatically, such as energy dispersion, band gap, carrier density, and current performance. $V_{G S}, L$, and $T_{\mathrm{OX}}$ variation and their effects on the $I_{D}-V_{D}$ characteristics were also analysed comprehensively. The results obtained show that quantum confinement effect is dominant at higher $V_{G S}$ and reduced $L$ and $T_{\text {OX }}$. From the above discussions, it is strongly suggested that the quantum effects must be taken into account in the design of uniaxial strained AGNRs.

\section{Conclusion}

In this paper, a unified $I_{D}-V_{D}$ characteristic of AGNRs under uniaxial strain has been developed. The modulation of $I_{D}-V_{D}$ characteristic due to the changes in the strain is found to be directly related to the modified electronic structure in the AGNRs channel region. While the introduction of strain imposes changes in the band gap and current values, the incorporation of quantum confinement effect results in dramatic reduction in the drain current performance. Quantum capacitance is greatly dependent on the magnitude of strain and ribbon width. The dependence of $I_{D}-V_{D}$ on applied gate voltage, channel length, and oxide thickness is highlighted and discussed. The discrepancies between the classical calculation and quantum calculation can be best explained by the threshold voltage shift and total gate capacitance degradation due to quantum confinement. The observed trends are consistenct with the recently reported simulation results. Compared to the previous works, this model offers a simplified approach of unified $I_{D}-V_{D}$ simulation and provides physical insights into the characterizations of uniaxial strained AGNRs.

\section{Conflict of Interests}

The authors declare that there is no conflict of interests regarding the publication of this paper.

\section{Acknowledgments}

The authors would like to acknowledge the financial support from the Ministry of Higher Education (MOHE), Malaysia, under the Fundamental Research Grant Scheme (FRGS) Project no. R.J130000.7823.4F146. They also thank the Research Management Centre (RMC) of Universiti Teknologi Malaysia (UTM) for providing excellent research environment to complete this work.

\section{References}

[1] Q. Xu, X. Duan, H. Liu, Z. Han, and T. Ye, "Low-cost and highly manufacturable strained-Si channel technique for strong hole mobility enhancement on 35-nm gate length pMOSFETs," IEEE Transactions on Electron Devices, vol. 54, no. 6, pp. 1394-1401, 2007.

[2] A. H. C. Neto, F. Guinea, N. M. R. Peres, K. S. Novoselov, and A. K. Geim, "The electronic properties of graphene," Reviews of Modern Physics, vol. 81, no. 1, pp. 109-162, 2009.

[3] J.-H. Wong, B.-R. Wu, and M.-F. Lin, "Strain effect on the electronic properties of single layer and bilayer graphene," The Journal of Physical Chemistry C, vol. 116, no. 14, pp. 8271-8277, 2012.

[4] M. F. Craciun, S. Russo, M. Yamamoto, and S. Tarucha, "Tuneable electronic properties in graphene," Nano Today, vol. 6, no. 1, pp. 42-60, 2011.

[5] H. Behera and G. Mukhopadhyay, "Strain-tunable band gap in graphene/h-BN hetero-bilayer," Journal of Physics and Chemistry of Solids, vol. 73, no. 7, pp. 818-821, 2012.

[6] D. V. Kosynkin, A. L. Higginbotham, A. Sinitskii et al., "Longitudinal unzipping of carbon nanotubes to form graphene nanoribbons," Nature, vol. 458, no. 7240, pp. 872-876, 2009.

[7] F. Guinea, "Strain engineering in graphene," Solid State Communications, vol. 152, no. 15, pp. 1437-1441, 2012. 
[8] N. Wei, L. Xu, H.-Q. Wang, and J.-C. Zheng, "Strain engineering of thermal conductivity in graphene sheets and nanoribbons: a demonstration of magic flexibility," Nanotechnology, vol. 22, no. 10, Article ID 105705, 2011.

[9] N. Rosenkranz, M. Mohr, and C. Thomsen, "Uniaxial strain in graphene and armchair graphene nanoribbons: an ab initio study," Annalen der Physik, vol. 523, no. 1-2, pp. 137-144, 2011.

[10] Y. Li, X. Jiang, Z. Liu, and Z. Liu, "Strain effects in graphene and graphene nanoribbons: the underlying mechanism," Nano Research, vol. 3, no. 8, pp. 545-556, 2010.

[11] K. Khaliji, M. Noei, S. M. Tabatabaei, M. Pourfath, M. Fathipour, and Y. Abdi, "Tunable bandgap in bilayer armchair graphene nanoribbons: concurrent influence of electric field and uniaxial strain," IEEE Transactions on Electron Devices, vol. 60, no. 8, pp. 2464-2470, 2013.

[12] J. Xia, F. Chen, J. Li, and N. Tao, "Measurement of the quantum capacitance of graphene," Nature Nanotechnology, vol. 4, no. 8, pp. 505-509, 2009.

[13] G. Kliros, "Effect of uniaxial strain on the current-voltage characteristics of graphene nanoribbon field-effect transistors," in Proceedings of the International Semiconductor Conference (CAS '13), vol. 2, pp. 27-30, Sinaia, Romania, 2013.

[14] M. Han, Y. Zhang, and H.-B. Zheng, "Effect of uniaxial strain on band gap of armchair-edge graphene nanoribbons," Chinese Physics Letters, vol. 27, no. 3, Article ID 037302, 2010.

[15] M. T. Ahmadi, Z. Johari, D. C. Y. Chek, N. A. Amin, and R. Ismail, "Modelling of graphene nanoribbon Fermi energy," Journal of Nanomaterials, vol. 2010, Article ID 909347, 6 pages, 2010.

[16] D. Gunlycke and C. T. White, "Tight-binding energy dispersions of armchair-edge graphene nanostrips," Physical Review B, vol. 77, no. 11, Article ID 115116, 2008.

[17] J. Guo, Y. Yoon, and Y. Ouyang, "Gate electrostatics and quantum capacitance of graphene nanoribbons," Nano Letters, vol. 7, no. 7, pp. 1935-1940, 2007.

[18] M. L. P. Tan, V. K. Arora, I. Saad, M. T. Ahmadi, and R. Ismail, "The drain velocity overshoot in an $80 \mathrm{~nm}$ metaloxide-semiconductor field-effect transistor," Journal of Applied Physics, vol. 105, no. 7, Article ID 074503, 2009.

[19] X. Zhou and K. Y. Lim, "Unified MOSFET compact I-V model formulation through physics-based effect transformation," IEEE Transactions on Electron Devices, vol. 48, no. 5, pp. 887-896, 2001.

[20] J. Kong and A. Javey, Carbon Nanotube Electronics, Springer, Boston, Mass, USA, 2009.

[21] M. T. Ahmadi, M. L. P. Tan, R. Ismail, and V. K. Arora, "The high-field drift velocity in degenerately-doped silicon nanowires," International Journal of Nanotechnology, vol. 6, no. 7-8, pp. 601-617, 2009.

[22] G. S. Kliros, "Modeling of carrier density and quantum capacitance in graphene nanoribbon FETs," in Proceedings of the International Conference on Microelectronics (ICM '10), pp. 236239, Cairo, Egypt, December 2010.

[23] K. Alam, "Uniaxial strain effects on the performance of a ballistic top gate graphene nanoribbon on insulator transistor," IEEE Transactions on Nanotechnology, vol. 8, no. 4, pp. 528-534, 2009.

[24] G. S. Jayadeva and A. DasGupta, "Compact model of shortchannel MOSFETs considering quantum mechanical effects," Solid-State Electronics, vol. 53, no. 6, pp. 649-657, 2009.
[25] M. A. Karim and A. Haque, "A physically based accurate model for quantum mechanical correction to the surface potential of nanoscale MOSFETs," IEEE Transactions on Electron Devices, vol. 57, no. 2, pp. 496-502, 2010. 

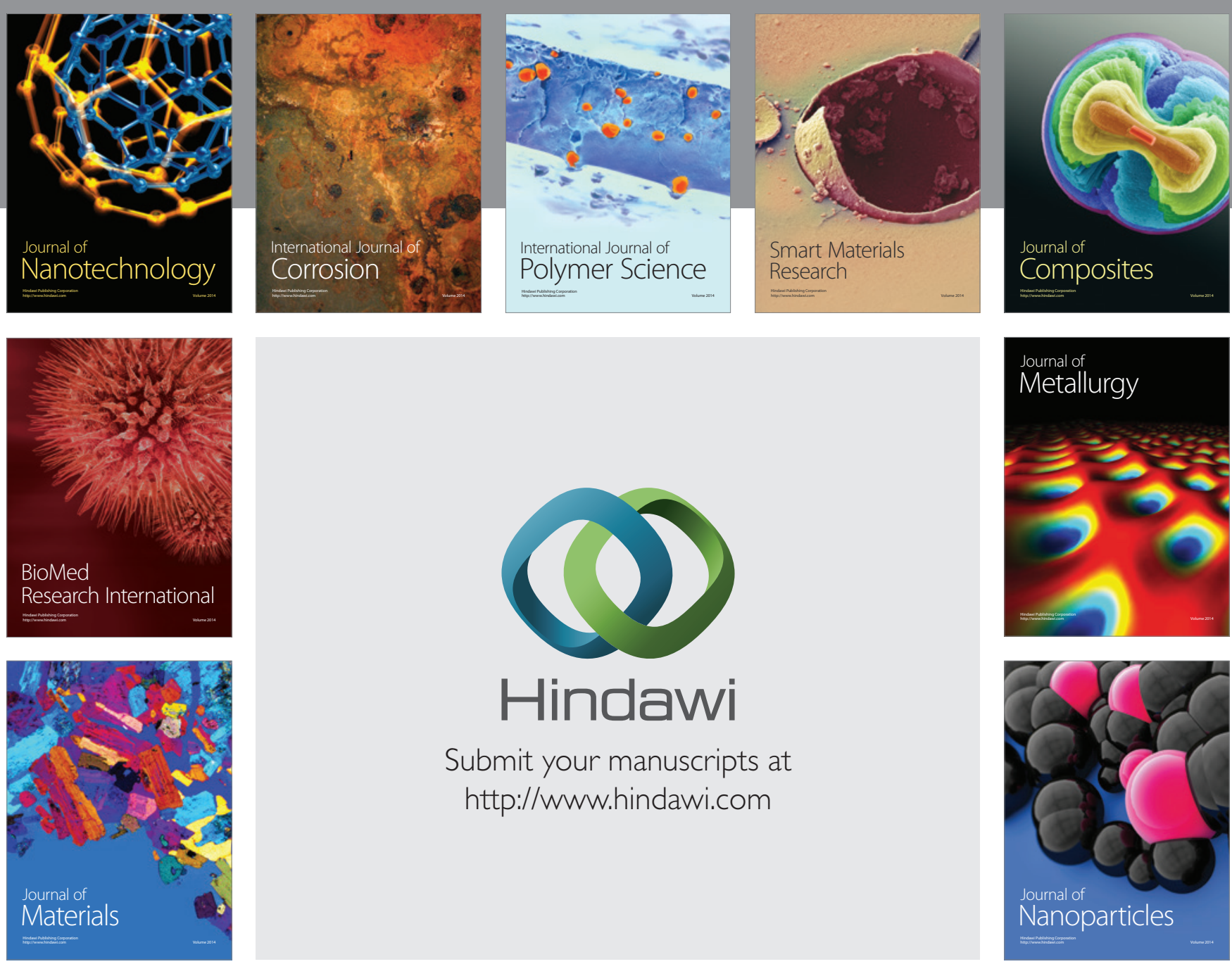

Submit your manuscripts at http://www.hindawi.com
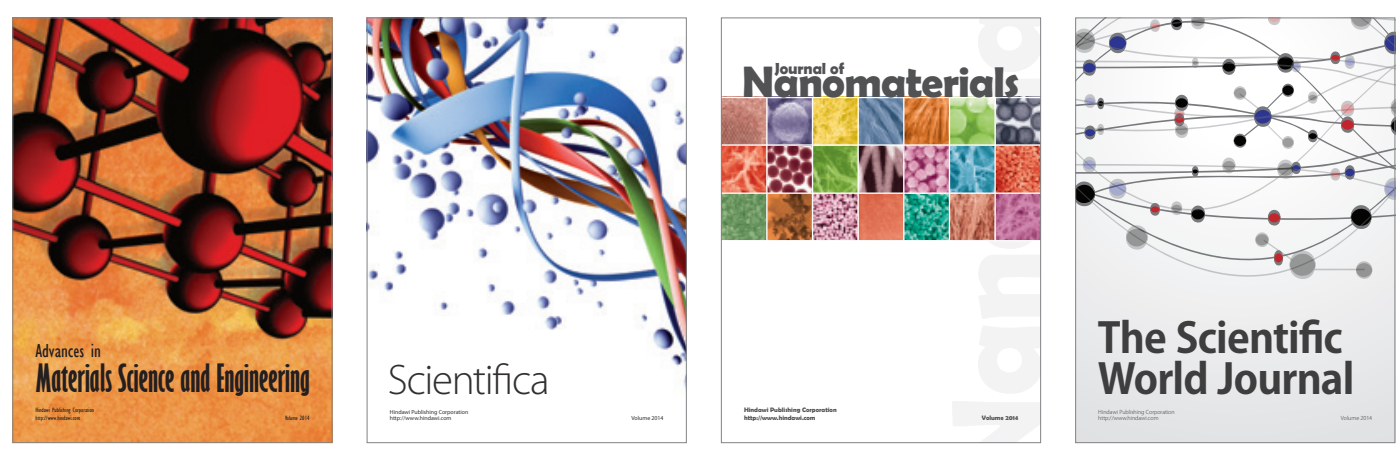

\section{The Scientific World Journal}
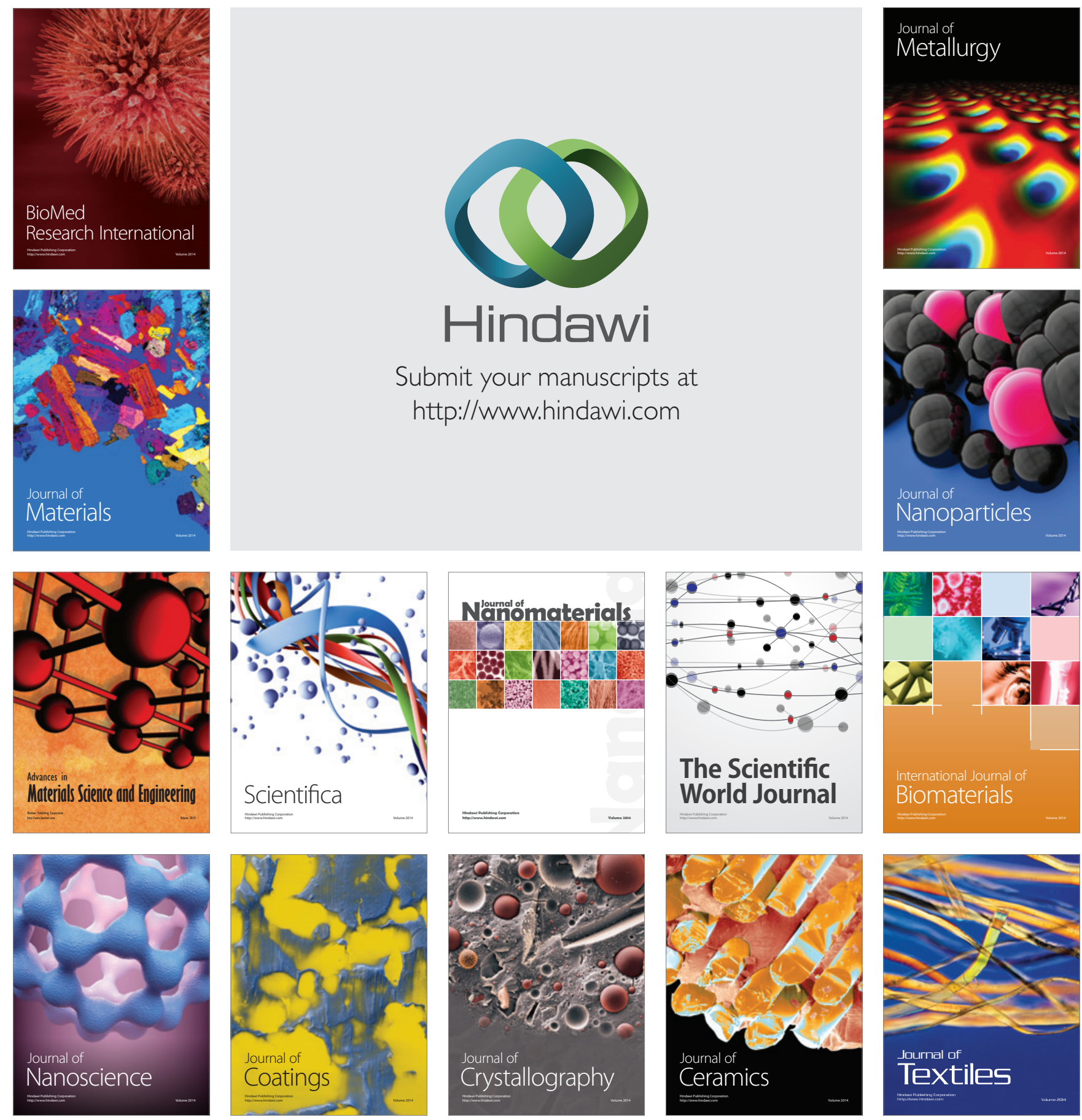